\title{
People are not coins: a reply to Hedden.
}

\author{
E. Viganò, University of Zurich \\ K. Hertweck, University of Zurich and Zurich University of Applied Sciences \\ C. Heitz, Zurich University of Applied Sciences \\ M. Loi, University of Zurich ${ }^{1}$
}

\begin{abstract}
:
This paper is a reply to "On Statistical Criteria of Algorithmic Fairness," by Brian Hedden. We question the significance of arguing that many group fairness criteria discussed in the machine learning literature are not necessary conditions for the fairness of predictions or decisions based on them. We show that it may be true, in general, that a statistical fairness constraint, $f_{n}$, is not a necessary condition for the fairness of all predictions (or decisions based on them). And yet, compatibly with this, most predictions or decisions involving people could be unfair if they violate $f_{n}$. We argue that a morally salient distinction exists between two types of predictions, human-individual-based vs. human-group-based predictions, and what may be necessary condition for the fairness of one type of prediction may not be a necessary condition for the fairness of the other.
\end{abstract}

\section{Introduction}

When making decisions, we often rely on probabilities. When betting whether a coin will land heads up, for example, we might calculate the probability of this happening and make our decision of whether and how much we want to bet on heads based on this probability. When deciding whether a hospital patient should be given a certain treatment, we might look at patients who have been assigned this treatment under similar conditions and calculate how often it cured the patient. From this, we can ascribe a probability of being cured to the current patient. Note that the data based on which we compute probability in the two cases is quite different. For the coin example, we can toss the coin as often as we want to in order to determine the probability of the coin landing heads up. In the medical example, we only get to treat the patient once. This means that we have to rely on the data of other patients for our probability estimate - we cannot treat the patient a thousand times and see what happens in each case.

In this essay, we therefore distinguish two kinds of calculations of probabilities, which lead to two kinds of probabilities, individual-based probabilities and group-based probabilities. Since we are concerned with decisions made about humans, we define these datasets and probabilities with respect to people and call them human-individual-based probabilities and human-group-based probabilities. We speak of human-individual-based probabilities if and only if the assessment of the probability is based only on data of that one person, or other things related to that person, and no other person is involved. We will shorten this term to " $h-$ individual-probability" in the rest of the text where the "h" is short for "human" and the "based"

\footnotetext{
${ }^{1}$ Corresponding author: michele.loi@uzh.ch.
} 
is left out for brevity. By contrast, we speak of human-group-based probabilities (h-group probabilities for short) if and only if the probability has been determined by taking data of other people into account (another person, at least). For example, one's chance of healing with a given therapy is estimated on the basis of how many people who were treated with the therapy were cured. We remain agnostic about whether this distinction matters from a purely epistemic point of view (i.e., whether it has implications about the strength of a probabilistic belief), or from a purely ontological point of view (i.e., whether h-individual probabilities and h-group probabilities refer to different properties of the world). We only claim that the distinction between h-individual probabilities and h-group probabilities matters from the moral point of view. If the distinction between h-individual and h-group probabilities is intelligible, then one can distinguish two types of practices in which decisions are taken about individuals based on probabilities assigned to them. We define h-individual practices as practices in which a decision maker decides something about person $\mathrm{P}$ on the basis of $\mathrm{h}$-individual probabilities assigned to $\mathrm{P}$. We define h-group practices as those in which the decision maker does the same on the basis of h-group probabilities assigned to $P$.

Hedden's recent paper on statistical criteria of algorithmic fairness ${ }^{2}$ provides good reasons to question whether some of the most widely considered statistical fairness principles (collectively labelled as the set F) are necessary conditions for the fairness of probability estimates. However, Hedden does not state clearly what kind of probability estimates - and thus practices, as defined above - he considers. We claim that while Hedden's argument is correct for $\mathrm{h}$-individual practices, it fails to show that any fairness constraint $f$ in the sent $\mathrm{F}$ is not a necessary condition of fairness, in the case of h-group practices. This paper provides an argument to this negative conclusion.

We illustrate the logic of our argument with an example. Suppose that someone claims that a whale is only healthy if it is blue. Blueness is thus claimed to be a necessary condition of whale health. Suppose we now learn that there are two kinds of whales: alpha-whales and betawhales. As it turns out, alpha-whales can also be healthy if they are red. Blueness is therefore not a necessary condition for their health. Since it is not a necessary condition for the health of alpha-whales, it is not a necessary condition for the health of whales in general. This finding, however, does not disprove that blueness is a necessary condition for the health of betawhales - it still could be, since we have not gained any new knowledge about beta-whales that would prove otherwise.

\footnotetext{
2 Brian Hedden, "On Statistical Criteria of Algorithmic Fairness," Philosophy \& Public Affairs 49, no. 2 (2021): 209-31, https://doi.org/10.1111/papa.12189.
} 


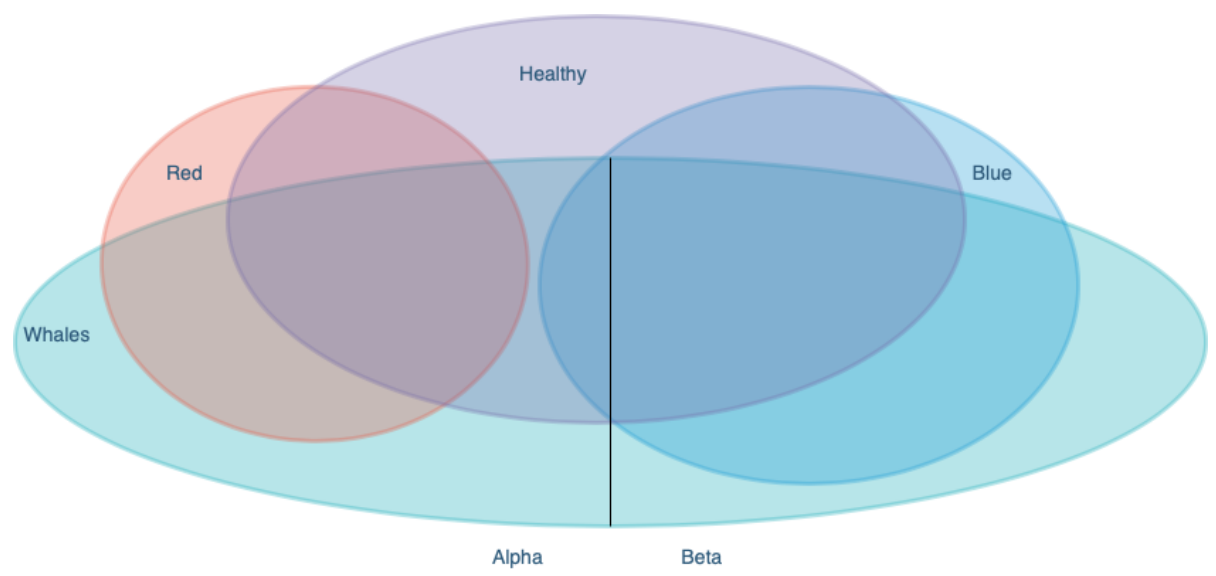

Figure 1. The whale set is dissected into two sub-sets (alpha and beta whales) by the straight line. These are two species of whales. Being blue is necessary for Beta whales to be healthy, but it is not necessary for Alpha whales to be healthy.

We are able to make a logical statement about whales in general - namely, being blue is not necessary for them to be healthy. This is certainly something useful to know, especially when confronting someone who seems unreasonably self-confident that, since some given whale is not blue (without investigating further if it is of the alpha- or beta-sub-kind), it must necessarily be sick. However, this should not lead anyone to disregard blueness as necessary for the health of whales, especially if beta-whales are much more common than alpha-whales.

Hedden's argument functions in a similar way in that it only proves that some fairness principles are not necessary for h-individual probabilities. However, he does not prove the same statement for h-group probabilities, which we claim are much more common and relevant in practice.

\begin{tabular}{|l|l|}
\hline Whales & Probability-based practices \\
\hline alpha whales & h-individual probability practices \\
\hline beta whales & h-group probability practices \\
\hline healthy & fair \\
\hline blue & some fairness constraint $\mathrm{f}_{\mathrm{n}}$ in $\mathrm{F}$ \\
\hline red & calibration \\
\hline
\end{tabular}

Table 1. Logical analogy between types of whales and types of probability-based practices.

We designate this paper as a reply to Hedden's paper. However, it is not a reply in the sense that we point out a problem or error in Hedden's argument. We do not challenge the conclusion about necessary conditions of probabilistic predictions or the resulting decisions in general. Our motivation is, rather, that people using h-group probabilities may erroneously take his conclusion as a good reason to believe that they may ignore the fairness constraints listed in $\mathrm{F}$ altogether. While Hedden's paper strictly speaking does not have that implication, we are worried that many readers may wrongly, due to a mistake of logic, conclude that it has.

Here we present a brief map of the paper. Section 2 summarizes Hedden's argument, to which our paper replies. Section 3 describes the logical structure of our argument. Section 4 explains the distinction between h-individual and h-group probabilities in more detail. Section 5 
describes h-individual and h-group practices, namely practices that rely on, respectively, hindividual and h-group probabilities. It anchors the theoretical discussion of decisions based on probabilities in actual practice of data science. Section 6 justifies the claim that the distinction between h-individual and h-group practices is morally salient. More precisely, we claim that $\mathrm{h}$-group practices could be seen as pro-tanto morally wrong because they violate the individual pro-tanto right of being treated as an individual. $\mathrm{H}$-individual practices are not vulnerable to this moral objection. Section 7 deals with the objection that our argument, understood as a defense of the possibility of the possible moral relevance of putative statistical fairness constraints in $\mathrm{F}$, offers one explanation too many of the unfairness of h-group practices, and thus it is self-undermining. Section 8 concludes the paper.

\section{Hedden's argument}

In his paper, Hedden describes an example in which a perfectly fair algorithm violates a number of common fairness metrics, i.e. the set $F$. We shall refer to the nth specific fairness constraint listed in that set as $f_{n}$. From that, Hedden infers for all $f_{n}$, that they are not necessary conditions for fairness, i.e., they do not have to be fulfilled by all prediction models that we call "fair".

We briefly summarize the example here, but refer the reader to Hedden's original paper for a more detailed discussion of the case. Hedden constructs a case in which people are assigned coins which can either land heads up or tails up. If a person's coin lands heads up, the person is deemed a "heads person". Each coin has a label which shows its probability of landing heads up. A predictor assigns risk scores to people meant to indicate their risk of being a heads person. This risk score is then used to predict the person as either being a heads person (risk score above 0.5 ) or a tails person (below 0.5 ). What the predictor does to assess the risk score is to simply to echo the label of the person's coin. This is an intuitively and perfectly fair approach. Hedden then brings in the idea of measuring fairness with respect to sociodemographic groups: In the example, people are sent to two different rooms, which represent the socio-demographic groups with respect to which we want to measure fairness. In one room, the coin labels are close to 0 and 1 , in the other room the labels are closer to 0.5 . This allows Hedden to construct a case with equal average coin labels. Nevertheless, due to the different distributions of labels, many fairness metrics are violated. These are the fairness metrics in F. (For a detailed demonstration of why they are violated, we again point the reader to Hedden's paper.)

The only metric that is fulfilled is calibration. In this case, calibration requires that for a given risk score, the ratio of people who have been assigned this risk score and in fact turn out to be heads people is roughly equal to this risk score. For the risk score 0.8 , for example, $80 \%$ of all the people who have been assigned this risk score should be heads people. This is clearly fulfilled if this is tested on enough people since a coin label of 0.8 means that it will land heads up in $80 \%$ of the cases. Hedden thus concludes that metrics in $\mathrm{F}$ cannot possibly be genuinely necessary. The only metric that can possibly be necessary (since it is still fulfilled in the perfectly fair coin example he constructed) is calibration.

\section{Outline of the reply}

The argument in this paper has the following form: 
$\mathrm{P} 1$. One can distinguish between two practices for determining ${ }^{3}$ probabilities to be assigned to persons: those that use data of other people, and those that only use data of the person to whom the probability is assigned. For brevity's sake we refer to these probabilities as humanindividual-based probabilities (shortly, h-individual probabilities), and human-group-based probabilities (shortly, h-group probabilities), respectively.

P2. Hedden's coin example is an instance of using a h-individual probability. So, he shows for any $f_{n}$ in $\mathrm{F}$ that $f_{n}$ is not necessary for the fairness of these probabilities in the h-individual practice Hedden examines. By way of implication, $f_{n}$ is not necessary for fairness in general.

P3. Many practices in data science involve h-group probabilities. This includes the COMPAS example that Hedden treats as paradigmatic.

P4. Practices involving h-group probabilities may differ from practices involving $h$-individual probabilities from the moral point of view.

P5. The fact that some $f_{n}$ is not a necessary condition for the fairness in general of probabilitybased practices does not logically exclude that $f_{n}$ may a necessary condition of the fairness of h-group probabilities practices.

Conclusion: Hedden has not offered an argument for data scientists using h-group probabilities to disregard $\mathrm{F}$ as a necessary condition for the fairness of their algorithms.

The argument for premise P5 has already been provided in the introduction, and is made more comprehensible by the whale analogy. The next sections provide support for the other premises: Section 4 will argue for P1 and P2, Section 5 for P3, and Section 6 for P4.

\section{Individual-based- and group-based-probabilities}

$\mathrm{P} 1$. One can distinguish between two practices for determining probabilities to be assigned to persons: those that use data of other people, and those that only use data of the person to whom the probability is assigned.

P2. Hedden's coin example is an instance of using a h-individual probability. So, he shows for any fn in $F$ that $f n$ is not necessary for the fairness of these probabilities in the $h$ individual practice Hedden examines. By way of implication, $\mathrm{fn}$ is not necessary for fairness in general.

Probabilities are used to deal with situations where there is uncertainty in the outcome. In the case of algorithmic decision making, where a decision maker wants to take a decision with respect to a human individual, we are dealing with the uncertainty of current or future features of persons (such as whether or not this person commits a crime). In Hedden's coin example, he associates a probability with each coin, "indicating its bias, or its objective chance of landing heads."

${ }^{3}$ Our usage of the term "determining" here refers to all processes that lead us to assign probabilities. This includes testing, which is necessary for the assignments to be considered scientifically justified. When using machine learning to assign these probabilities, the term refers to the entire machine learning pipeline, i.e., data collection, data cleaning, training, testing etc.

${ }^{4}$ Hedden, "On Statistical Criteria of Algorithmic Fairness," 219. 
While bracketing all philosophical considerations about the meaning of the concept "probability," we assume that there is something like an objective chance $x$ of landing heads which is given for each individual coin, as Hedden suggests. So, we can actually associate this probability $x_{i}$ with each individual $i$, in the sense of a feature of this individual. Actually, many prediction-based decision algorithms make exactly this kind of association, and the associated probability is taken as an input for the decision, as Hedden suggests for his "perfectly fair and unbiased predictive algorithm."

A frequent problem in practice, however, is that it is often not possible to determine such a probability based on the data of a single individual, or it is extremely costly to do this. Take, for example, the probability that a 50-year old individual will die of cancer within the next 10 years, which would be a typical prediction task for a predictive decision algorithm. This probability depends on the complete genetic predispositions of this individual, as well as on everything that has happened in the life of this individual during the last 50 years, and maybe even more. Since there was never a person on earth with the same genotype and the same life experiences, it is impossible to determine the probability by considering only that individual, even if we had access to all the information about it.

In the case of simple systems such as coins, the standard way of determining probabilities for a specific outcome to be predicted - such as landing heads - is to repeat some experiment many times: we throw the coin in a well-defined manner under well-defined environmental conditions many, many times and count how often it lands heads or tails, respectively. Under the assumption that the coin does not change its nature during these observations and afterwards (stationarity), we can then determine the probability of landing heads up to an arbitrary precision. After this is done, we can treat this probability as a feature of the coin, and label it accordingly. It is easy to see that this is indeed a feature of this specific coin, as only this coin is used to determine its value.

However, for most events that are relevant in data-based prediction of individuals, there is just not enough history to observe enough repetitions. We cannot observe enough lifetime of an individual for determining their probability of committing a crime in the next month to any reasonable accuracy, letting alone the obvious violation of the stationarity assumption. Furthermore, as the example of the 50-year old individual above shows, repetitions over a lifetime are often just not possible. ${ }^{6}$

In such cases, however, another approach may be chosen. We combine observations of different individuals for determining the searched-for probability. For example, we may observe a sample of 100050 -year old people over a period of 10 years and record how many of them die during this period. The percentage of deceased people is then interpreted as a probability, and we assign all individuals of the group this probability. In fact, this probability is extremely useful because it does allow us to make predictions: If we take a new group of 50year old persons, selected according to the same selection procedure as the first group, we

\footnotetext{
5 Hedden, 219.

${ }^{6}$ We do, however, rely on individual histories when making informed guess about individuals we know. For example, we may think it probable that a friend who has been very often late to appointments in the past will be late in the future. These can be described as intuitive probabilistic beliefs, but are not formalized as applications of statistical methods.
} 
can predict the expectation value of how many of them will die, together with confidence intervals (under some additional assumptions).

For the coin example, this approach means: instead of repeating a coin throw experiment 1000 times with the same coin, we take 1000 identical coins and throw each once, recording on which side the coin lands. Statistical theory tells us that taking the number of observed heads and dividing them by 1000 yields a non-biased estimate of the true probability of each of these coins - assuming that all coins do have the same probability (which is meant by "identical coins").

A similar approach is used in the training phase of practically any data-driven prediction algorithms: observations of events of numerically distinct but similar individuals are analyzed together, and the resulting estimated probability is then assigned to each of those individuals. ${ }^{7}$ This is done in all cases where repetitions are not possible, but also in many other cases, where collecting data of different individuals over a short time period is just easier, cheaper, or more convenient than observing single individuals over a long period (and then assuming that the recorded past can be extrapolated to predict the future).

Thus, for determining the searched-for probability, we can either apply a practice that involves only data (observations) of the individual under consideration, or a practice that also involves data of other individuals. As explained before, for humans, we call the so-determined probabilities human-individual-based probabilities (h-individual probabilities) and humangroup-based probabilities (h-group probabilities), respectively. More generally, also when not referring to humans, we may speak of individual-based probabilities and group-based probabilities. But the general distinction is not morally salient the way - we shall argue - the human distinction is. In the case of deciding what to do with our coins, for example, the distinction between individual-based and group-based probabilities is not morally important.

As clarified in Section 6 below, our ground for distinguishing these different ways of determining probabilities is not epistemological or ontological, but moral. Our focus here is not on the logic, epistemology, or ontology of probabilities. Our argument depends on identifying an especially morally problematic type of probabilities. Group-based probabilities are not always morally problematic: it is, for example, not morally problematic to assign a probability to a coin on the basis of a comparison with other identical coins and to act on the basis of that assessment. The only case in which group-based-probabilities are problematic is the one where the data of different human individuals are grouped for determining a probability for a single person. This explains the need to distinguish h-group probabilities from generic groupbased probabilities and $\mathrm{h}$-individual probabilities from generic individual based-probabilities.

It is now easy to show that in Hedden's coins example, the risk scores assigned to people ${ }^{8}$ are $\mathrm{h}$-individual probabilities. Hedden starts with "coins of varying biases", ${ }^{9}$ making clear that the bias is a generic property of the coin itself. Accordingly, the coin can be labelled with this

\footnotetext{
${ }^{7}$ In practice, this is a bit more complicated. Prediction models assume some kind of smoothness or functional dependency and then interpolate the training data.

${ }^{8}$ Hedden talks about people being "heads people" in Hedden, "On Statistical Criteria of Algorithmic Fairness," 222.

${ }^{9}$ Hedden, 217.
} 
probability. Then he states: "each individual in the population is randomly assigned a coin;"10 this means that the probability assigned to each person (which is the probability that her coin lands heads) does not depend in any way on anything related to other people. In such a case, Hedden's statement that this assignment is "perfectly fair and unbiased"11 is true. We will explain in Section 6 and 7 why this may not be as obvious in the case the probability is a hgroup probability. But before doing that, we will show that the case of h-group probability is very common (it is the most common species of whale, according to our allegory), because many algorithmic practices involve h-group probabilities.

\section{Probability in machine learning}

P3. Many practices in data science involve h-group probabilities. This includes the COMPAS example that Hedden considers to be paradigmatic.

Let us now turn our focus to machine learning and discuss what kinds of probabilities we encounter in this field. There are different subfields of machine learning, but what is relevant for our paper is the usage of machine learning to make predictions based on input data. This is what the subfield of supervised machine learning is concerned with. For this, a data set consisting of input features $x$ and target variables $y$ exists. This could, for example, be data about individuals who have in the past been granted a loan by a given bank. Each row in this data, i.e. each accepted loan application, comes with a label $y$, which is the target for the prediction task. If the goal in the banking example is predicting whether a given applicant will repay their loan on time, y would be a label for just that: whether the individual repaid their loan on time. The goal of supervised machine learning is to find a mapping from input features $x$ to target variables $y$.

To do this, a large enough data set needs to be provided. This data is typically split into two parts: a training set and a testing set. An algorithm "trains" on the training data set, meaning it infers rules about the relationship of $x$ and $y$ from the training data. The algorithm defines what these rules might look like. In logistic regression, for example, a sigmoid curve is fit to the data. The "rules" would then correspond to the weight assigned to the different input features. Other machine learning algorithms might fit other curves or models to the training data. The algorithm keeps adjusting these rules until the predictions appear to work well for the training data. Finally, the rules it found are tested on the test set to ensure generalizability.

The data set of input features $x$ and target variables $y$ can consist both of data from a single individual or many different individuals. In the case that the data set consists of data from a single individual, we speak of an h-individual practice. In the case that the data set consists of data from more than one individual, we speak of an h-group practice. The advantage of $h$ group practices is that the data collection process is easier and faster. Moreover, the prediction model of h-group practices is more generalizable. With enough data, such a model could, for example, be applied to any individual - as opposed to the individualized models which are expected to only be valid for a single individual. More importantly, however, for a lot of cases, it is simply impossible to train a model on the data of only one individual. For this reason, $\mathrm{h}$ group practices are extremely common in data science. This includes the COMPAS example

\footnotetext{
10 Hedden, 219.

11 Hedden, 2019.
} 
that Hedden specifically refers to. In the case of COMPAS, data from thousands of defendants who were admitted for bail were collected along with the target label y of whether they arrested within the next two years. This target variable $y$ is supposed to represent recidivism. Here, it would clearly be not only inefficient, but also impossible to build a machine learning model based on only a single individual's data. The developers of the tool therefore trained their model based on data of many defendants. The case thus represents an instance of an h-group practice.

Hedden states that he is "not claiming that the case of people, coins, and rooms is realistic or completely analogous to cases like COMPAS. Of course, it is not."12 He then goes on to explain several reasons for why the coin example is not completely analogous to COMPAS, but "idealizes away from these complications."13 What he does not appear to consider though is the question of whether the probability labels of coins are comparable to the probability outputs of COMPAS and what influence this comparison has on the claims he makes. Our argument is that the coins' probabilities and the defendants' assigned probabilities are different in such a way that the claims Hedden proves for the coin example are merely applicable for cases of h-individual practices, but not for h-group practices, such as COMPAS, which are very common in machine learning.

\section{The moral difference between human-individual-based practices and human-group-based practices}

P4. Practices involving h-group probabilities may differ from practices involving hindividual probabilities from the moral point of view.

When comparing h-individual and h-group practices, a prima facie moral difference emerges. It is the intuition that there is something inherently wrong with predicting a person's behavior based on data of other persons deemed to be "similar" (h-group probabilities), instead of only using data of the considered person, e.g. the person's past behavior and her features based on individual features. For example, one may predict that a friend will be most likely late, if that friend has often been late in the past, and that a friend will be most likely punctual, if that friend has normally been punctual in the past. The strength of the belief that the friend will be late could then be expressed as a h-individual probability. Let us provide a different example. A lender may lend money to you at a time of difficulty, and expect you to be able to repay it, while denying the same loan to another creditor with a similarly low income and needing the loan just as much. The lender may simply ask you to produce a proof of your past savings and that your future earnings will be equal or higher and ask the other person to produce a proof of her savings and current or future earnings, and conclude that you are more likely than her to save money to repay the debt and interest in the future. These individualized predictions do not feel morally problematic in the way h-group practices are, such as predicting that someone is less likely to repay a debt because he or she is a woman, a minority member, a foreigner, a person who does not own a car, or the member of a specific income group, or any combination of these features, whenever some statistical association exists that would justify

\footnotetext{
12 Hedden, 223.

13 Hedden, 224.
} 
this inference epistemically. Because of such a prima facie distinction, we cannot simply assume that practices generating or employing h-individual and h-group probabilities are morally analogous; we rather need to delve into the intuition that $\mathrm{h}$-individual and $\mathrm{h}$-group practices are morally different due to their different ways of estimating probabilities about people.

In moral philosophy, there is a moral principle $\mathrm{M}$ that grounds the intuition that there is a moral difference between $\mathrm{h}$-individual and h-group probabilities. This principle $\mathrm{M}$ is: one should treat people as individuals. We may have an intuitive idea about what principle $\mathrm{M}$ requires, but the latter is not self-evident. The understanding of principle $M$ requires a clarification of what we mean by "individual", when this term is attributed to persons. In the principle M, a person's individuality concerns her being that particular person and not another one, namely her being a unique individual. The individual here is necessarily a person, i.e., the subject of a special regard.

After having clarified the meaning of "individual," when the latter is applied to persons, we need to specify what the right attitude towards this individual is, namely how to treat a person properly, where "properly" means in a way that is appropriate to what this individuality is. We shall use the expression "respect for individuality," here, to indicate that mode of treatment that is compatible with treating people as individuals. It is not essential for this argument to determine what this expression amounts to exactly. But it seems plausible that, whatever it means, it cannot mean treating the individual based on the assumption that she will act similarly to how other people have acted who are similar to that person in some respect. For this intuitively amounts to treating the individual as a specimen of a more general category or group.

In legal studies and moral philosophy, several authors considered the principle of treating people as individuals as a morally legitimate obligation whose violation brings about some moral wrongness. In the legal field, principle $M$ is discussed with regards to the value of statistical evidence for legal proof in courtrooms. The main issue in this field is the weight of statistical evidence for proving a defendant guilty. ${ }^{14}$ Several legal scholars contended that statistical evidence cannot ground verdicts because using statistical evidence to form a judgment about a defendant violates principle $\mathrm{M} .{ }^{15}$

14 Sarah Moss, Probabilistic Knowledge, Probabilistic Knowledge (Oxford University Press, 2018), https://doi.org/10.1093/oso/9780198792154.001.0001.

15 Adrian A. S. Zuckerman, "Law, Fact Or Justice," Boston University Law Review 66 (1986), https://heinonline.org/HOL/Page?handle=hein.journals/bulr66\&id=493\&div=\&collection=; David T. Wasserman, "The Morality of Statistical Proof and the Risk of Mistaken Liability," Cardozo Law Review 13 (1991), https://heinonline.org/HOL/Page?handle=hein.journals/cdozo13\&id=957\&div=\&collection=; Amit Pundik, "Freedom and Generalisation," Oxford Journal of Legal Studies 37, no. 1 (July 2016): gqw016, https://doi.org/10.1093/ojls/gqw016. Others defend the opposing view, i.e. using statistical evidence is not necessarily a failure to treat the defendant as an individual, where principle $M$ is conceived as respecting people's autonomy. See Federico Picinali, "Base-Rates of Negative Traits: Instructions for Use in Criminal Trials," Journal of Applied Philosophy 33, no. 1 (February 2016): 6987, https://doi.org/10.1111/japp.12109; Mike Redmayne, "Exploring the Proof Paradoxes," Legal Theory 14, no. 4 (2008): 281-309, https://doi.org/10.1017/S1352325208080117. 
In moral philosophy, several scholars have dealt with principle $M$ in the context of statistical discrimination, ${ }^{16}$ racial profiling ${ }^{17}$ - which is an instance of statistical discrimination -, and stereotyping. ${ }^{18}$ According to Eidelson, principle $\mathrm{M}$ is a moral requirement that demands treating the individual as partly the results of her previous choices with which she determined her life. ${ }^{19}$ Lippert-Rasmussen conceived principle $M$ as the demand to make accurate judgments about a person, using all the relevant information reasonably available to one. ${ }^{20}$ Beeghly contended that principle $\mathrm{M}$ in the forms provided by Eidelson and Lippert-Rasmussen can be considered a moral obligation "sometimes or always." 21 Moss considered profiling as an epistemic failure that has a moral implication, ${ }^{22}$ which is the violation of an interpretation of principle M: the rule of consideration. ${ }^{23}$ This is a moral norm stating that, in forming beliefs about a person, one should consider the possibility that this person, as an individual, can be an exception to statistical generalization deriving from statistical evidence. ${ }^{24}$ According to Basu, beliefs about a person based on statistical evidence violate the moral demand of relating to others as people, which is her understanding of principle $\mathrm{M} .{ }^{25}$ Similarly, in Blum, ${ }^{26}$ principle $M$ is understood as a fundamental form of acknowledgment of persons, whose violation is a moral fault. ${ }^{27}$

${ }^{16}$ Benjamin Eidelson, "Treating People as Individuals," in Philosophical Foundations of Discrimination Law, ed. Deborah Hellman and Sophia Moreau (Oxford: Oxford University Press, 2013); Kasper LippertRasmussen, "We Are All Different': Statistical Discrimination and the Right to Be Treated as an Individual," Journal of Ethics 15, no. 1-2 (June 2011): 47-59, https://doi.org/10.1007/s10892-010-90956; Kaspar Lippert-Rasmussen, Born Free and Equal? A Philosophical Inquiry into the Nature of Discrimination (Oxford: Oxford University Press, 2014), chap. 11, https://doi.org/10.1093/analys/anv063.

17 In profiling, one forms or acts on beliefs based on statistical evidence about a trait or behavior of a person. In racial profiling, one forms or acts on beliefs based on statistical evidence about a person's ethnicity.

${ }^{18}$ Lawrence Blum, "Stereotypes and Stereotyping: A Moral Analysis," Philosophical Papers 33, no. 3 (2004): 251-89, https://doi.org/10.1080/05568640409485143; Erin Beeghly, "Failing to Treat Persons as Individuals," Ergo, an Open Access Journal of Philosophy 5, no. 26 (July 2018): 687-711, https://doi.org/10.3998/ergo.12405314.0005.026.

${ }^{19}$ Eidelson, "Treating People as Individuals," 216.

${ }^{20}$ Lippert-Rasmussen, "'We Are All Different': Statistical Discrimination and the Right to Be Treated as an Individual," 54. Lippert-Rasmussen however conceives the distinction as being between statistical vs. non-statistical information. He uses " non-statistical information" for "information gained through X's perception of $Y$, other people's testimony about $Y$ delivered to $X, X$ 's memory of what $Y$ did in the past, available to $X "$ " (p. 51). He does not consider the possibility of statistical information derived from a single individual, e.g., counting how many times someone has been late or punctual in the past.

${ }^{21}$ Beeghly, "Failing to Treat Persons as Individuals," 708.

22 Moss, Probabilistic Knowledge, 221.

${ }^{23}$ Moss, 223.

${ }^{24}$ Moss, 221.

${ }^{25}$ Rima Basu, "What We Epistemically Owe To Each Other," Philosophical Studies 176, no. 4 (2019): 928, https://doi.org/10.1007/s11098-018-1219-z.

${ }^{26}$ Blum, "Stereotypes and Stereotyping: A Moral Analysis."

${ }^{27}$ Blum, 272-73. 
The scholars that defended principle $\mathrm{M}$ as a moral requirement did not argue that is an absolute moral principle. It is considered a pro tanto principle that can be overridden by other moral obligations that are relevant in the context of action, ${ }^{28}$ disregarded in case of limited or no individual evidence (i.e., information about the individual person), ${ }^{29}$ and not required in fleeting interactions with people. ${ }^{30}$

As principle $\mathrm{M}$ is a pro tanto principle, its violation is morally permissible - even required -, when the benefits of holding a belief grounded on statistical evidence overcome the harm deriving from treating somebody just as a member of a group, or when holding this belief keeps society safe. For instance, an emergency room doctor who has to decide the best treatment for a person is morally required to violate principle $M$, if the use of statistical evidence based on the person's ethnicity for choosing the treatment increases the chance to save this person's life. ${ }^{31}$ Similarly, if statistical evidence shows that a person of a specific gender and age is more likely to have committed a crime, police are justified in treating people with such characteristics as just members of these reference classes. Moreover, the scarce availability of information about the individual person makes the violation of principle M permissible. When getting information about an individual is costly or not possible, one is all-things-considered morally justified to base one's belief on that individual on statistical evidence derived from other people's data. ${ }^{32}$

On the other hand, some scholars in moral philosophy did not consider principle $\mathrm{M}$ as a moral requirement and its violation as morally wrong. ${ }^{33}$ According to Arneson and Levin, ${ }^{34}$ principle $M$ is not morally required as using statistical evidence to form a belief about a person is not (even pro tanto) morally wrong. According to Schauer, ${ }^{35}$ principle M may not even be initially plausible. He contended that the difference between treating people individually and based on statistical evidence is not clear. This is because treating people on the basis of individualized evidence requires assuming some generalizations that ground the individualized treatment on statistical evidence ${ }^{36}$ In reply, it is true that using even individualized evidence requires

${ }_{28}$ Moss, Probabilistic Knowledge, 224; Basu, "What We Epistemically Owe To Each Other," 927; Lippert-Rasmussen, "We Are All Different': Statistical Discrimination and the Right to Be Treated as an Individual," 57.

${ }^{29}$ Lippert-Rasmussen, "'We Are All Different': Statistical Discrimination and the Right to Be Treated as an Individual," 54; Eidelson, "Treating People as Individuals," 224.

${ }^{30}$ Blum, "Stereotypes and Stereotyping: A Moral Analysis," 282.

${ }^{31}$ Moss, Probabilistic Knowledge, 224.

${ }^{32}$ Lippert-Rasmussen, "'We Are All Different': Statistical Discrimination and the Right to Be Treated as an Individual," 54; Eidelson, "Treating People as Individuals," 224.

33 Richard J. Arneson, "What Is Wrongful Discrimination," San Diego Law Review 43 (2006), https://heinonline.org/HOL/Page?handle=hein.journals/sanlr43\&id=799\&div=\&collection=; Michael Levin, "Responses to Race Differences in Crime," Journal of Social Philosophy 23, no. 1 (March 1992): 5-29, https://doi.org/10.1111/j.1467-9833.1992.tb00481.x; Frederick F. Schauer, Profiles, Probabilities, and Stereotypes (Cambridge-London: Harvard University Press, 2003).

34 "Responses to Race Differences in Crime," 23.

${ }^{35}$ Schauer, Profiles, Probabilities, and Stereotypes, 19.

${ }^{36}$ Schauer, 101, 103, 172. Schauer's argument concerns the plausibility of distinguishing, with respect to principle M, between beliefs grounded in statistical evidence and beliefs grounded in evidence of a different form. The moral distinction we invoke is not the one between statistical vs. non-statistical evidence but the one between two forms of statistical knowledge. While we agree with the need for 
assuming some statistical generalization. But as the case of throwing a single coin a thousand times shows, it is not necessary in general for a statistical generalization to consider individuals other than the individual the probability is assigned to. Thus, contra Schauer, it may indeed be conceptually possible to only treat individuals on the basis of statistical generalizations that do not violate principle $\mathrm{M}$.

The lack of agreement on whether principle $\mathrm{M}$ is a moral requirement does not undermine our statement that $\mathrm{h}$-individual and $\mathrm{h}$-group practices are prima facie morally different. Therefore, we do not argue for the existence of an ultimate moral difference between h-individual and hgroup practices, i.e., a view that people have reasons to hold after careful reflection. We rather contend that the problem is still open. No ultimate rejection of this view has been generally acknowledged by experts in moral philosophy. This makes it reasonable not to disregard principle $\mathrm{M}$ as a currently viable hypothesis explaining why h-group probabilities are pro-tanto wrong. What could follow from the possible moral truth of $\mathrm{M}$ may have important implications for the debate on statistical fairness constraints.

Now that we have identified and explained the principle $M$, we can see in detail in which sense it traces a moral difference between $\mathrm{h}$-individual and h-group practices. H-individual probabilities are estimated on the basis of data related to the person on which the prediction is made. These data include the decisions made by the person, the actions taken, and other individual features, etc. A h-individual probability is thus estimated on the basis of what the person did (being late or punctual, thrifty or spendthrift) or other characteristics (financial independence, stability of employment, having dependent children) that do not require comparisons with the outcomes of other people to reasonably inform a guess about the future outcomes of that person. But usually, in data science, the information that is used to make predictions on a person is not based on this person's individual data. By contrast, h-group probabilities are estimated on the basis of data of other people, not of the person about whom the prediction is made. $\mathrm{H}$-group probabilities are founded on an attribution of the person to a specific group, sharing the same or similar aspects with that person (e.g., being a woman, a bank clerk, a member of the same age group). In h-individual practices, the person is treated as an individual because $\mathrm{h}$-individual practices use $\mathrm{h}$-individual probabilities, which are estimates in which only observations about that individual person are considered. $\mathrm{H}$-individualand h-group practices are morally different because the former practices, even when they rely on statistical generalizations, are not treating the person as an instance of a more general type. The degrees of belief are not informed by generalizations from other individuals, but simply by observations about that person's own past and the generalizations that can be drawn from them.

In conclusion, the authors that defend principle $\mathrm{M}$ as a moral requirement contend that it requires that our judgments about persons are constrained by the concept of persons as unique beings. Since it is a pro tanto principle, it is not a prohibition to use statistical evidence tout court, nor an obligation to eschew generalization. Such a theoretical position thus provides support to two propositions of our approach to h-individual and h-group practices: $(1)=(M)$ : it

inductive generalizations in our handling of individualized evidence, we observe that inductive generalizations can also be based on observing the behavior of a single individual over time, so they do not have to necessarily rely on comparisons with similar individuals. The example of throwing a coin one thousand times vs. throwing a thousand coins one time makes the general conceptual distinction comprehensible. 
is pro tanto wrong not to treat people as individuals; (2) making decisions about people based on h-group probabilities is pro tanto wrong because it violates the principle $\mathrm{M}$.

\section{The "one-explanation too many" objection.}

The argument of Section 6 shows that h-group-practices could be pro tanto wrong because they violate the principle $\mathrm{M}$ that people ought to be treated as individuals. Suppose that the wrongness in question is a case of unfairness. As this suffices to make h-group-practices unfair, it may seem that any further necessary condition for the fairness of predictions, $f_{\mathrm{n}}$, that applies only to h-group-practices, would be redundant. For we know already that h-grouppractices are unfair, qua h-group-practices, hence, we ought not to engage with them. What is the point, then, of claiming that they are unfair because they violate $F_{n}$ ? How could $F_{n}$ be necessary for something to be fair, if these practices are already unfair?

Another way to state this objection is by considering the role that $F_{n}$ plays in contrast arguments. As Shelly Kagan explains:

The contrast strategy clearly assumes that if a factor has genuine moral relevance, then for any pair of cases, where the given factor varies while others are held constant, the cases in that pair will differ in moral status. (The difference, of course, need not be so extreme as to make, e.g., one act permissible and the other act impermissible; the point is simply that some difference in moral status should exist.) $)^{37}$

In application to this case, the contrast strategy applies as follows:

Premises:

I. A given $n$th statistical fairness definition, $f_{\mathrm{n}}$, is a necessary condition of the fairness of $\mathrm{h}$ group practices.

II. H-group practices are pro-tanto unfair.

III. Here is a practice, $\mathrm{P}$, which uses h-group probabilities.

IV. P is unfair, all things considered (from II and III, and the fact that no overriding consideration obtains that makes $P$ fair, all things considered.)

$V$. If $f_{\mathrm{n}}$ is a necessary condition for $\mathrm{P}$ being fair, then, when $\mathrm{P}$ violates $f_{\mathrm{n}}$, is it unfair, for that reason.

VI. P violates $f_{\mathrm{n}}$.

VII. $\mathrm{P}$ is unfair (from I, III, and V, and VI).

37 Shelly Kagan, "The Additive Fallacy," Ethics 99, no. 1 (October 1, 1988): 12 , https://doi.org/10.1086/293033. 
VIII. $f_{\mathrm{n}}$ being violated does not make a difference in moral status. (From the identity of IV and VII.)

Conclusion: $f_{\mathrm{n}}$ has no genuine moral relevance.

This argument, however, commits the additive fallacy, namely to assume that in all, or at least most cases if $f_{\mathrm{n}}$ has genuine moral relevance, it must make a difference in moral status. ${ }^{38}$ Instead, $f_{n}$ may have genuine moral relevance and only make a difference in moral status in some cases, but not all. Before we present a case in which $f_{n}$ makes a difference in the fairness of a h-group practice, we describe a more familiar and concrete case that has some similarities and prepares the reader for our case.

Suppose, for argument's sake, that judging individuals based on the color of their skin is always pro tanto wrong, meaning, wrong unless an overriding moral reason for doing that exists. Now, consider a case in which judging individuals based on the color of their skin (e.g., using ethnic identity information in the search of a terrorist) is necessary to prevent a very sever wrong to be committed, e.g., a nuclear bomb to be detonated. ${ }^{39}$ Surely, while the use of skin color information can be wrong pro tanto (and hence call for apologies to the innocent individuals examined), the action may be morally permissible all things considered, even obligatory. The utilitarian value of the action, it is at least conceivable, may override the reason that makes it pro-tanto wrong, and make the action right all things considered.

Now let us turn to a similar case involving h-group probabilities. Consider a case in which $\mathrm{P}$ involves using h-group probabilities as the basis of a decision. Given principle $M$, this is protanto unfair. Suppose now that $P$ enables us to prevent a more serious moral wrong, and using h-group probabilities raises the probabilities of success from $60 \%$ to $80 \%$. The utilitarian argument may contribute to a justification of h-group probabilities being fair, all things considered, in this case.

Now, let us suppose for the sake of the argument, that drastically improving the probability of preventing a more serious wrong is not sufficient to make $P$ fair. In order for $P$ to be fair, all things considered, the misclassification harm imposed on individuals of different groups must also be proportionate, whatever that means exactly. ${ }^{40}$ This is not a prima facie implausible view, especially when the technical means to make it proportionate (e.g., some algorithm guaranteeing that specific statistical criteria be satisfied) exist, and if experts could agree on a definition of what "proportionate" means. ${ }^{41}$

\footnotetext{
${ }^{38}$ Kagan, "The Additive Fallacy."

${ }^{39}$ Mathias Risse and Richard Zeckhauser, "Racial Profiling," Philosophy \& Public Affairs 32, no. 2 (2004): 131-70, https://doi.org/10.1111/j.1088-4963.2004.00009.x.

${ }^{40}$ Risse and Zeckhauser.

${ }^{41}$ This is a possible way of reformulating Clinton Castro's view about this problem that makes it not only fully compatible with this view, but also its direct ancestor. Castro argues that "if x's treatment of $y$ exposes $\mathrm{y}$ to a risk of being misclassified that $\mathrm{y}$ could reasonably reject, then $\mathrm{x}$ fails to respect $\mathrm{y}$ 's individuality" Clinton Castro, "What's Wrong with Machine Bias," Ergo, an Open Access Journal of Philosophy 6 (2019): 423, http://dx.doi.org/10.3998/ergo.12405314.0006.015. It seems that the substance of Castro's argument is compatible with an alternative conclusion, namely "if x's treatment of $\mathrm{y}$ exposes $\mathrm{y}$ to a risk of being misclassified that $\mathrm{y}$ could reasonably reject, then, even if $\mathrm{x}$ fails to respect y's individuality, the treatment is fair to x". The fulfilment of a fairness constraint $f n$ would then specify the misclassification risks that all the different groups involved cannot reasonably reject.
} 
It is, then, logically possible for one of the statistical fairness criteria $f_{n}$, in $\mathrm{F}$, e.g., equalized odds, to be a necessary condition for the fairness of using h-group probabilities in this case and, perhaps, all other cases involving h-group probabilities. The statistical criterion $f_{n}$ makes a moral difference here, because $\mathrm{P}$ is pro tanto unfair and can be fair all things considered by virtue of the utilitarian argument, but only if $f_{n}$ is satisfied. Satisfying $f_{n}$ could, indeed, be what makes the misclassification harm imposed on different groups proportionate. We are not claiming that experts know already what that $f$ is. But clearly Hedden's discovery pertaining to an instance of h-individual probability does not offer any reason for experts to exclude any of the putative fairness constraints included in $\mathrm{F}$.

To repeat, the following two claims are not contradictory:

(1) all practices involving $h$-group probabilities are (all things considered) fair only if proportionate attention to different groups is ensured; a given statistical criterion $f_{n}$ may specify what "proportionate" means in all contexts where h-group probabilities are involved. For all we know, the criterion $f_{n}$ may be a member of $\mathrm{F}$.

(2) there is one possible use of $H$-individual probabilities which is fair, even it violates $f_{n}$. Thus, $f_{n}$ is not a necessary condition for the fairness of all predictions (or the decision practices based on them).

It is absolutely clear that, from a purely logical point of view, (2) does not provide any reason to doubt (1).

Now suppose someone objected that $f_{n}$ has no genuine moral relevance if it only applies to $h$ group practices. For we can already infer that all h-group practices are unfair, being violations of $\mathrm{M}$. This may be correct in some sense. But inferring from this that $f_{n}$ cannot be a genuine fairness constraint for all h-group practices amounts to committing the additive fallacy. ${ }^{42}$ As Kagan has shown, it is wrong to believe that, if $f_{\mathrm{n}}$ is a morally relevant consideration for some practice $\mathrm{P}$, it must influence the moral status of all h-group practices. The constraint $f_{n}$ can (logically speaking) be a necessary condition for the fairness of all h-group practices and yet, the satisfaction of $f_{n}$ may only make a morally salient difference when utilitarian arguments provide good reasons for a specific $P$ to be used, where $P$ would otherwise be unfair. Hedden's argument cannot provide a proof of the contrary.

\section{Conclusion}

In our paper, we distinguish h-individual and h-group probabilities. We do not claim that generic individual and generic group probabilities are morally, epistemically, or ontologically different, e.g., that any interesting difference exists between a coin bias identified by throwing the same coin 1000 times, and one obtained by throwing 1000 identical coins once. We only claim there is at least a prima facie moral difference between computing a probability based on data of other persons deemed to be "similar" (h-group probabilities), instead of only using data of the considered person, e.g., how the person behaved in the past (h-individualprobability). While a 2-euro coin can easily be replaced by another 2-euro coin, people are seen as unique. When it comes to humans, there may be a moral difference between using data from other people in support of a general rule allowing us to infer something about an individual person vs. using data only from that individual in support of an analogous inference.

42 Kagan, "The Additive Fallacy." 
This moral difference may make h-group probabilities morally problematic. If so, using them could require a moral justification, which is not required in a parallel case involving only hindividual probabilities. Hedden's conclusion about probability practices in general is thus compatible with any $f_{n}$ in $\mathrm{F}$ being a necessary condition for the fairness of h-group practices, but not for h-individual practices.

Because of this, we claim that Hedden's result may not be too significant in practice. What may not be practically significant is showing that some statistical fairness constraint $f_{n}$ is not a necessary condition of fairness in general. This may simply follow from the fact that it is not a statistical fairness constraint in those rare cases in which h-individual probabilities are used. Such cases - cases in which $f_{n}$ is not a necessary condition for fairness - may turn out to be the exception rather than the rule. For, as discussed in Section 5, h-group probabilities are very common in the world of data science, especially when making predictions about people. If what we have argued is correct, the arguments Hedden provides against caring about whether any fairness metric $f_{n}$ of specific set criticized in his article (such as equalized odds) are in practice not relevant for most data scientists. For all we know, those fairness metrics could still be valid options for them and there could be important moral arguments against their violation. 